\title{
Visibilidade e monitoramento científico na área nuclear e ciências relacionadas: uma perspectiva a partir da produtividade do IPEN-CNEN/SP
}

Adilson Luiz Pinto

Doutor em Documentação. Professor do Departamento de Biblioteconomia da UFMT. Especialista em indicadores de C\&T e Redes Sociais

Mery P. Zamudio Igami

Mestre em Ciencia da Informação. Bibliotecária do IPEN CNEN SP

José Carlos Bressiani

Doutor em Ciencia dos Materiais,. Professor no curso de Pós-graduação em Tecnologia Nuclear do IPEN CNEN SP

O objetivo deste trabalho foi monitorar e obter indicadores sobre a atividade científica desenvolvida no IPEN-CNEN/SP, visível internacionalmente. Utilizou-se como parâmetro os artigos de periódicos registrados na base de dados Web of Science (WoS), nas três últimas décadas. Identificou-se o núcleo de revistas e de autores, o índice de visibilidade, o de colaboração, bem como o crescimento científico. Para análise dos dados, utilizou-se as técnicas bibliométricas e cienciométricas. Pelos resultados obtidos é possível afirmar que o IPEN tem representatividade e visibilidade significativa na WoS.

Palavras-chave: Produção científica; Indicadores bibliométricos; Artigos científicos; Visibilidade; Energia nuclear. 


\section{Visibility and scientific surveillance in the nuclear area and related sciences: a perspective from the productivity of the IPEN-CNEN/SP}

The main objective of this paper was to monitor and obtain indicators concerning the international visibility of the scientific activities developed in IPEN-CNEN/SP. The articles published along the three decades and registered in the Web of Science (WoS) were used as parameters. It was possible to identify the central journal and author nucleus, index of visibility and collaboration as well as the scientific growth. Scientometric and Bibliometric instruments were used for data analysis. The results obtained confirms a significative IPEN visibility in WoS.

Keywords: Scientific production; Bibliometric indicators; Scientific articles; Visibility; Nuclear energy.

\section{Considerações iniciais}

A produção do conhecimento científico está fortemente associado à pesquisa, a qual segue princípios metodológicos para que a validade dos seus resultados sejam assegurados. Os resultados das pesquisas se materializam em forma de vários produtos, entre eles incluí-se a produção científica. Para um maior entendimento do processo de produção científica apresentam-se alguns conceitos, como o de Witter (1997), afirmando que a produção científica é uma expressão que engloba processos e produtos distintos, bem como pessoas, associações, agências financiadoras e seus múltiplos consumidores. Por outro lado, Lourenço (1997, p. 1) define produção científica como...

[...] toda produção documental, independente do suporte desta - papel ou meio magnético - sobre um determinado assunto de interesse de uma comunidade científica específica que contribua para o desenvolvimento da ciência e para abertura de novos horizontes de pesquisa.

Em Campanário (2004), a alegação de produção científica vai além da definição, onde a mesma é derivada de um importante revolucionário descobrimento, que não pode ser considerado definitivo até que não abandone o laboratório onde foi realizado e não seja comunicado ao resto da comunidade científica, representando a figura do cientista que a sociedade caracterizou. Porém, este mesmo cientista já não tem espaço 
em suas atividades isoladamente, como também era figurado em outras épocas.

Campanário (2004) continua afirmando que o trabalho científico é um empenho coletivo que está sempre sujeito à comprovação de outros membros da comunidade científica e que os processos de comunicação em ciência são fundamentais para o seu desenvolvimento.

Na comunicação científica destaca-se o trabalho de Garvey (1979), com o elucidativo título de Comunicação: a essência da ciência, no qual afirma que a comunicação científica inclui todas as atividades associadas com a produção, disseminação e o uso da informação, a partir do momento em que o pesquisador tem a ideia investigativa e vai até que a informação sobre os seus resultados seja aceita como constituinte do conhecimento cientifico.

A rapidez na divulgação faz com que descobertas sejam colocadas à disposição de outros pesquisadores, no menor espaço de tempo possível, permitindo que o conhecimento circule e alimente outras pesquisas, mantendo o fluxo da informação e a retroalimentação da ciência.

Em complemento, o surgimento e o amplo uso das tecnologias da informação propiciaram ainda mais a interação e colaboração entre pesquisadores de localidades mais remotas, com trocas de informações e discussões de resultados de forma virtual e em tempo real. O ambiente eletrônico é sem dúvida um meio ilimitado de oportunidades, que contribui significativamente para a construção e divulgação do conhecimento científico.

Segundo Macias-Chapula (1998) publicar os resultados de suas pesquisas é um compromisso que os cientistas são compelidos a cumprir. O avanço do conhecimento produzido pelos pesquisadores tem que ser transformado em informação acessível para a comunidade científica e, assim, a pesquisa é desenvolvida num contexto de troca.

Várias são as razões que levam um pesquisador a publicar sua produção intelectual: salvaguardar a propriedade intelectual, divulgar resultados pesquisados, ser reconhecido pela comunidade científica, melhorar o seu status intelectual, obter ascensão profissional e manter identificação com os pares que realizam pesquisas similares (MOURA, 1997). Depreende-se, então, que comunicar a descoberta científica é um ato inerente e imprescindível para a atividade científica e, consequentemente, para o progresso da ciência.

Neste sentido, o periódico científico é o principal canal formal utilizado na comunicação científica. Os artigos publicados nestes periódicos são a forma definitiva de publicação dos resultados de uma investigação.

Independente do formato adotado para sua publicação, a função do periódico cientifico define-se como memória e arquivo do conhecimento, meio de comunicação entre membros de diversas comunidades científicas e, certamente, é a formalização do conhecimento (BIOJONE, 2001). 
Como meio de preservação e disseminação do conhecimento científico favorece a leitura e a citação do trabalho por outros pesquisadores.

A importante função do periódico científico é mais evidente naquelas publicações que adotam a avaliação pelos pares (peer review), a qual demonstra a preocupação em verificar a validade do método utilizado no desenvolvimento de uma pesquisa e a qualidade dos resultados alcançados.

Os pesquisadores mais produtivos ganham prestígio e reconhecimento pelos seus pares. Publicar em periódicos reconhecidos internacionalmente, indexados em bases de dados de renome, significa conseguir algo além de sua certificação como pesquisador, mas sim garantir a propriedade e prioridade do objeto da pesquisa (MIRANDA; PEREIRA, 1996).

A institucionalização da ciência, o crescimento das comunidades científicas e principalmente a explosão de artigos publicados em periódicos motivaram o interesse em avaliar a qualidade do material publicado. No processo de avaliação são utilizados os indicadores bibliométricos, entre outros, gerados com base nos artigos científicos publicados em periódicos considerados de qualidade internacional e que refletem a produção científica de uma determinada comunidade. Os indicadores bibliométricos de atividade científica têm sido utilizados nos últimos anos como importante ferramenta na gestão de política científica e, neste caso, serão vislumbrados de forma a averiguar o monitoramento e a visibilidade da produtividade do IPEN frente à Web of Science (WoS), bem como o princípio da publicação científica.

\section{Objetivos}

Os estudos bibliométricos, aplicados à produção científica de uma instituição, constituem-se em um dos melhores instrumentos de gestão; os seus resultados subsidiam e fundamentam as ações voltadas a tomada de decisão. Portanto, o objetivo deste trabalho é obter indicadores sobre a atividade científica desenvolvida no Instituto de Pesquisas Energéticas e Nucleares (IPEN-CNEN/SP), visível internacionalmente, utilizando como parâmetro os artigos de periódicos publicados na base de dados Web of Science, nas três últimas décadas.

O estudo enfoca dois pontos cruciais: (i) a visibilidade, para obter grau de importância do IPEN dentro do cenário internacional e sua conduta dentro da Web of Science; e (ii) o monitoramento da produtividade dos autores que representam o nome do IPEN.

Especificamente, os dados serão analisados quantitativamente (produção) e qualitativamente (impacto) com a finalidade de:

1. descrever numericamente a sua evolução ao longo do período; 
2. conhecer o Fator de Impacto (FI) e o núcleo dos periódicos utilizados para comunicação dos trabalhos (segundo o modelo de Bradford);

3. verificar o índice- $h$ dos agentes do IPEN;

4. identificar as redes dos autores em relação às suas colaborações e da instituição (IPEN) para com outras instituições do seguimento no Brasil e no estrangeiro;

5. aplicar as métricas (sociométrica) para verificar a intensidade com que os autores constroem suas colaborações e parcerias, identificando assim, a centralidade $^{1}$ de colaborações e densidade ${ }^{2}$ destas relações, representadas por grau, vetores ${ }^{3}$, intermediação ${ }^{4}$ e aproximação ${ }^{5}$ destas parcerias (PINTO; RODRÍGUEZ-BARQUÍN, 2005).

\section{Marco teórico}

Assim, dentro dos objetivos propostos, apresenta-se brevemente a contextualização do assunto abordado, definições dos índices utilizados bem como uma apresentação da área estudada, na qual será enfatizada a bibliometria e sua função, a cobertura para análises de citações e os fatores de impacto dos periódicos, juntamente com a área que será explorada, no caso, a de energia nuclear.

\subsection{A bibliometria}

Tague-Sutcliffe (1992) define bibliometria como sendo o estudo dos aspectos quantitativos da produção, disseminação e uso da informação explícita. A bibliometria desenvolve padrões e modelos matemáticos para medir esses processos, utilizando os seus resultados para elaborar previsões e apoiar tomadas de decisões.

Spinak (1998) afirma que não é possível interpretar os dados sobre as necessidades informacionais e seu uso sem reconhecer que os

\footnotetext{
1 Pode ser calculada de acordo com diferentes medidas, proporcionando diferentes conceitos de centralidade. A forma mais simples e intuitiva de medir a centralidade é através do grau dos pontos do grafo (FREEMAN, 1978). Um ponto é central se está bem conectado com os demais pontos em seu entorno. As medidas de centralidade baseadas no grado podem ser consideradas medidas de centralidade local (NIEMINEN, 1974).

2 É um conceito da rede social relacionado à quantidade de linhas (ou conexões) que mantém interligado um conjunto de pontos. Quanto mais conexões (linhas) existir numa rede, mais densa ela será.

${ }^{3}$ É o ente matemático que reúne em si módulo, direção e sentido. Todo segmento que apresenta essas três características pode representar um vetor.

${ }^{4}$ É uma medida do número de vezes que um nodo aparece no caminho mais curto entre outros dois nodos. $O$ índice de intermediação é a soma dos quocientes entre o número de caminhos geodésicos que unem dois nodos e o número de vezes que passam pelo nodo em questão. A intermediação fornece uma aproximação do peso como conector do nodo e sua importância é essencial para manter unida a rede.

${ }^{5}$ É a soma das distâncias que separam um nodo do resto dos demais nodos da rede e aproxima seu peso.
} 
pesquisadores são os elementos centrais dos muitos sistemas que abordam cada aspecto do seu trabalho, entre os sistemas mais importantes nos quais estão envolvidos: o ambiente cultural e político, os colégios invisíveis, as organizações formais, os grupos de trabalho, o sistema legal e econômico, as associações profissionais e, finalmente, os sistemas de informação. A bibliometria é, portanto, uma disciplina de alcance multidisciplinar. É aquela que analisa um dos aspectos mais relevantes e objetivos desta comunidade.

Dentro das técnicas de bibliometria uma das mais conhecidas é a lei de Bradford, também denominada "lei de dispersão".

De acordo com esta lei, se os periódicos científicos forem colocados em ordem de produção decrescente de artigos pertinentes, relacionados com um determinado assunto, esses periódicos poderão ser divididos em zonas de produtividade, onde a primeira zona constitui o núcleo de periódicos mais estreitamente relacionados com a área que está sendo estudada. A segunda zona deve conter o mesmo número de artigos do núcleo, porém, o número de títulos será maior e assim sucessivamente, na proporção de $1: \mathrm{n}: \mathrm{n}^{2} \ldots$ (NORONHA et al., 1978; BENCE; OPPENHEIM, 2004).

Ainda de acordo com essa lei, os artigos de interesse para os pesquisadores podem ser publicados não apenas em um núcleo de periódicos relativos ao assunto da sua especialidade, mas também em outros títulos de áreas relacionadas. A atual tendência de multidisciplinaridade da ciência corrobora o enunciado da lei de Bradford. A crescente multiautoria dos artigos é uma clara tendência desta afirmação. Cada vez mais os pesquisadores comunicam-se e relacionamse entre si, não somente em nível nacional como também em nível internacional, desenvolvendo pesquisas em parceria, os chamados colégios invisíveis.

\subsection{Fator de impacto}

O Institute for Scientific Information (ISI), localizado na Filadélfia (EUA) publica e atualiza, periodicamente, a fonte de informação Journal Citation Report (JCR) com o objetivo de fornecer dados quantitativos para subsidiar a avaliação dos títulos de periódicos. É importante lembrar que o ISI somente analisa e fornece o ranking dos periódicos indexados nas bases de dados da Web of Science, produto do ISI que contempla as áreas de ciências naturais, sociais e artes/humanidades (Science Citation Index, Social Science Citation Index e Arts and Humanities). O fator de impacto (FI) é uma das medidas mais conhecidas no meio científico para a avaliação de revistas, utilizada pelo ISI e também por outros institutos e bases de dados, como a experiência do SciELO e do Scopus.

O JCR é um instrumento de avaliação criado pelo ISI, para formar um ranking dos periódicos científicos, indexados nas bases do WoS, baseado no Fator de Impacto. Fornece também outros dados sobre cada título de periódico, como meia-vida das citações e o índice de imediatez. 0 
fator de impacto é o resultado do número de citações (C) que um periódico recebe em dois anos, dividido pelo número de todos os artigos neles publicados, neste mesmo período (A). Portanto, seu cálculo é realizado pela fórmula: $\mathrm{FI}=\mathrm{C} / \mathrm{A}$. Sua função é auxiliar na avaliação da importância do periódico, principalmente quando comparado com outros títulos da mesma área.

\section{3 Índice de citação}

Semelhante ao fator de impacto em revistas, o índice de citação contempla a projeção de autores, revistas e documentos consultados por terceiros.

A citação é o meio mais comum de atribuir créditos e reconhecimento para aqueles cujos trabalhos têm contribuído para o desenvolvimento das ideias em diferentes campos. O artigo de periódico com a sua lista de referências citadas é provavelmente, e assim permanecerá, o meio universalmente aceito pelo qual a instituição científica registra e divulga os resultados de suas investigações.

Com o surgimento de índices de citação comerciais, nas últimas três décadas, a importância das citações ganhou uma nova dimensão na vida dos cientistas, departamentos de pesquisa, institutos e universidades (MACIAS-CHAPULA, 1998).

Este mesmo autor comenta que a indexação de citações está fundamentada na premissa de que uma citação bibliográfica é a expressão de uma relação entre dois documentos, aquele que cita e aquele que é citado.

Neste sentido é necessário pensar na citação como um processo. Os resultados desse processo seriam as listas de citações que acompanham os trabalhos acadêmicos. O tipo e a composição dessas listas refletem a personalidade do autor e seu meio profissional. Frente a essas ações permanecerá, sempre, um desconhecimento entre as razões que levam um autor a citar outro, e é este aspecto da citação que os sociólogos reivindicam como sendo o seu campo de pesquisa.

Os sociólogos argumentam que as citações somente podem ser compreendidas por meio do exame das condições sociais que predispõem os cientistas a citar da maneira como fazem, assim sendo, para compreender o significado da citação é necessário entender a sua realidade social.

Outro índice relacionado a citações é o índice $h$, que começa a ganhar espaço no meio científico, mesmo tendo divergências de sua aplicabilidade. Este índice se baseia no maior número de trabalhos que tem um autor pelo mesmo número de citações e pode ser aplicado para saber quantos documentos esse autor tem, em evidência, no meio acadêmico/científico. O índice $h$ foi desenvolvido por J. E. Hirsch (2005), um pesquisador da Universidade da Califórnia e, para melhor compreensão recorre-se a um exemplo: 
- Busca-se em uma base de dados ou em um motor web (como Google Acadêmico) todos os artigos "Leite, BA" e ordena-se pelo número de citações recebidas;

- Na continuação, listam-se os primeiros resultados (que são os que interessam para a análise), tal como segue abaixo, documentos versus o índice de citações recebidas:

Artigo 1.- número de citações (27)

Artigo 2.- número de citações (26)

Artigo 3.- número de citações (25)

Artigo 4.- número de citações (12)

Artigo 5.- número de citações (8)

Artigo 6.- número de citações (7)

Artigo 7.- número de citações (5)

Neste exemplo, o índice $h$ desse autor seria (h 6), porque é o máximo de artigos que possuem o mesmo número de citações. Para ter um índice $h$ de 7 aparições ou mais, o autor deveria receber, ao menos, o mesmo número de citações que o posto de ordem ocupado na lista de artigos.

\subsection{Apresentação da área estudada}

Diante de todas as concepções dos estudos métricos e suas vertentes é fundamental se inserir o objeto de estudo, onde estas técnicas serão aplicadas.

A área nuclear e ciências relacionadas, objeto deste estudo, são bastante recentes no país. Pode-se considerar que o início de suas atividades de pesquisa se remonta à década de 1950, com uma função estratégica para o desenvolvimento de tecnologia de ponta no segmento energético brasileiro, onde um dos principais agentes neste setor é o Instituto de Pesquisas Energéticas e Nucleares (IPEN) .

Fundado em 1956, o IPEN está localizado no campus da Universidade de São Paulo (USP), e sua missão enfoca "a melhoria da qualidade de vida da população brasileira, produzindo conhecimentos científicos, desenvolvendo tecnologias, gerando produtos e serviços, formando recursos humanos nas áreas nuclear e ciências correlatas".

O IPEN é uma autarquia estadual, vinculada à Secretaria de Desenvolvimento do Governo do Estado de São Paulo, gerido técnica e administrativamente pela Comissão Nacional de Energia Nuclear (CNEN), dessa forma vinculando-se ao Ministério de Ciência e Tecnologia do Governo Federal e associado à USP, para fins de ensino e pesquisa (PósGraduação Stricto Sensu).

O desenvolvimento de atividades do IPEN abrange desde as pesquisas de novos radiofarmacos até a sua produção, em regime comercial. Também se destacam os programas de pesquisas e desenvolvimentos em outras áreas, como nas aplicações das radiações ionizantes, na ciência e tecnologia nuclear, nos reatores nucleares e ciclo 
dos combustíveis, nas energias renováveis, nos materiais e nanotecnologia, na tecnologia lasers e na biotecnologia.

Além das atividades de pesquisa, produção e serviços o IPEN é uma instituição que difunde os seus conhecimentos por meio do ensino de Pósgraduação, nos programas de Tecnologia Nuclear, Mestrado Profissionalizante e disciplinas optativas para a graduação em cursos da USP (IPEN, 2008).

Atualmente, existem oito cursos de Pós-Graduação na área nuclear no país, todos sob a função do CNEN, dos quais sete estão concentrados na região sudeste e um na região nordeste. Eles foram avaliados pela CAPES, com as seguintes especificações:

- três cursos com nível 3;

- dois cursos com nível 4;

- dois cursos com nível 5; e

- um curso com nível 6.

Entre esses oito cursos de Pós-Graduação, encontra-se o de São Paulo, avaliado com nível 6, ministrado no IPEN-CNEN/SP, local onde foi desenvolvido este estudo.

\section{Metodología}

Trata-se de uma pesquisa de natureza qualitativa/quantitativa, onde foi utilizada a metodologia dos estudos métricos (bibliometria e análise de redes sociais), caracterizada como uma análise temporal do tipo documental e de abordagem exploratória, transcorrendo no período de 1967 até 2007.

A fonte de dados utilizada para este estudo foi a versão on-line das principais bases de dados da Web of Science - WoS (Science Citation Index Expanded, Social Science Citation Index; Arts \& Humanities Citation Index), publicada pela empresa Thomson Scientific Information, considerada uma das bases científicas multidisciplinares mais abrangentes, com rigorosos critérios de qualidade.

O estudo enfoca, especificamente, os artigos publicados em periódicos de âmbito internacional, produzidos pelos pesquisadores do instituto em questão.

Conforme comentado anteriormente, um dos objetivos deste trabalho é verificar a visibilidade científica da Instituição IPEN, para tanto a primeira ação foi efetuar uma pesquisa pelo nome da Instituição, IPEN-CNEN/SP - no campo ADDRESS, nas bases de dados da WoS.

Logo no início do estudo, foi observado que a falta de padronização do nome da instituição foi um inconveniente limitante, que teve que ser contornada apriori, para obter resultados mais realísticos. Assim, após várias tentativas, alternando o nome da instituição na estratégia de busca na WoS, foi obtido um resultado não coerente quando confrontado com os números apresentados pela base de dados sobre a produção científica 
mantida pela Unidade de Informação do IPEN. Essa base de dados local é depositária de toda a produção científica da instituição, desde sua criação, em 1956. Portanto, serviu como parâmetro para determinar o número, senão exato, bastante próximo do real.

Após uma detalhada operação de verificação e consistência dos dados, obteve-se um total de 1927 artigos, publicados em periódicos internacionais, registrados na WoS e confrontados com a base local do IPEN.

Um estudo realizado somente por uma instituição, como é o caso, deve ter um parâmetro de comparação. Para isto foi decidido utilizar os índices de produção científica de outras duas instituições congêneres, tanto em nível nacional como internacional. No caso, recorreu-se também a (i) produtividade do Instituto Nacional de Pesquisas Espaciais (INPE), localizado em São José dos Campos, São Paulo, por ter características de instituição de pesquisa e ensino e modelo administrativo semelhantes ao IPEN; e a (ii) Australian Nuclear Science \& Technology Organisation ANSTO, que é atuante na área nuclear em nível internacional e que possui uma estrutura e área de atuação muito similar a do IPEN.

Em relação à recuperação dos dados, estipulou-se uma estrutura única, com um delimitador através do nome das instituições pesquisadas em várias formas, justamente porque os recursos de busca e recuperação de informação da WoS não padroniza regularmente esse quesito. Foi o mecanismo viável que se encontrou para tentar evitar ruídos e nomes similares.

Definidos o processo de busca e recuperação e o número de artigos com o qual era factível trabalhar, procederam-se as análises bibliométricas.

O tratamento dos dados foi realizado em duas escalas. Na primeira, foi definida uma nomenclatura (download*) própria para os dados serem visualizados no CiteSpace (CHEN, 2006), para a análise de colaborações e para serem vislumbradas suas estruturas e campos dentro do ProCite (SAXTON, 2001), que executa uma divisão dos campos e determina a normalização de autores e de títulos de revistas. Para a segunda escala foi executada uma conversão dos dados em formato TXT e tabulações simples de adequação ( $\wedge$ t para $\wedge p$ ) para permitir a visualização dentro da base em Access (MICROSOFT, 2007a) e planilhas de cálculos simples em Excel (MICROSOFT, 2007b).

Em relação aos dados estatísticos para verificar a importância dos pesquisadores da própria instituição em variáveis, como núcleo de revistas e de autores, índice de visibilidade, índice de colaboração e crescimento científico, utilizou-se as técnicas bibliométricas e cienciométricas.

Para identificar $o$ núcleo de revistas mais utilizado pelos pesquisadores do IPEN em relação à publicação de seus trabalhos, aplicou-se o Modelo matemático de Bradford (1934), chamada Lei de Dispersão, por meio do qual foi determinado o núcleo das revistas mais produtivas, multiplicado pelo fator de proporcionalidade de títulos entre as zonas. Nesta análise se calcula a soma por ranking do maior ao menor, 
em três dimensões. O cálculo é uma divisão do total por 3, formando uma zona de grande porcentagem (1 ${ }^{a}$ escala), uma outra zona de mediana porcentagem representativa ( $2^{a}$ escala) e uma terceira zona de pequena porcentagem representativa ( $3^{a}$ escala), $\mathrm{p}: \mathrm{p} 1: \mathrm{p} 2: 1: \mathrm{n}: \mathrm{n}^{2}$.

Para a análise da identificação dos autores mais produtivos, utilizouse o modelo matemático de Price (1973). Usou-se a somatória dos autores que publicaram na WoS, obtendo um ranking do maior para o menor, determinando a população de maior representatividade dos autores, onde o número total de artigos foi elevado à raiz quadrada, delimitando assim, a elite dos autores.

Para obtenção do índice de visibilidade foram utilizados os padrões da teoria de Platz, que representa a presença de revistas ou autores em seu contexto científico, sendo visualizada como o número total de citações que os autores recebem, dividido pelo número total de artigos publicados por uma revista. Nesse caso, utilizou-se as revistas em que os principais autores do IPEN publicaram, no período estudado.

Em relação ao grau de colaboração definiu-se a porcentagem total de documentos com autoria múltipla, classificada como co-ocorrência e se realizou um índice de grafos $^{6}$ (visualização das relações em gráficos e sua representação Sociométrica), recuperando-se os índices de periodicidade dos autores, centralidade (FREMAN, 2000) e o índice de vetores da rede (BATAGEL]; MRVAR, 2003), tanto de relações diretas como indiretas.

Para determinar o crescimento científico, utilizou-se um índice de elevação por ano da instituição estudada, enfocando o modelo de distribuição exponencial (ESCUDER VALLES; MURGUI IZQUIERDO, 1995) (PRICE, 1973), demonstrando o comportamento da instituição no período estudado.

\section{Resultados e Discussões}

Para a consolidação dos resultados, considerou-se importante verificar o desempenho científico do IPEN frente ao seu crescimento exponencial, entretanto, a análise tornou-se mais elucidativa quando se estabeleceu uma comparação com as outras instituições selecionadas, para conhecer a dinâmica da atividade científica no setor estudado. $\mathrm{Na}$ FIG.1 é possível visualizar esse crescimento em comparação ao desempenho de outra instituição brasileira, o INPE, e outra australiana, a ANSTO.

O período de estudo foi distribuído em quinquênios (1978/1982; 1983/1987; 1988/1992; 1993/1997; 1998/2002; 2003/2007), porém,

\footnotetext{
${ }^{6}$ São artefatos matemáticos que permitem expressar de uma forma visual muito simples e efetiva as relações que se dão entre elementos de diversas índoles. Um grafo simples está formado por dos conjuntos: (i) conjunto $\mathrm{V}$ de pontos chamados vértices ou nós; e (ii) um conjunto de pares de vértices, que se chamam aristas ou arcos e que indicam que nodos estão relacionados. De uma maneira mais informal podemos dizer que um grafo é um conjunto de nodos com enlaces entre eles, denominados aristas ou arcos - que são as linhas dos gráficos.
} 
optou-se por manter o primeiro período com uma abrangência maior, 1967/1977 (11 anos), por reunir uma quantidade de produção de artigos científicos menores que os demais períodos quinquenais, justificando sua agregação. Na comparação do crescimento científico entre as três instituições destaca-se o desempenho do INPE, mesmo tendo uma constante semelhante a partir do quinquênio de 1988/1992, diferente da proporção apresentada pela ANSTO que saltou de 10 artigos (1983/1987) para 211 artigos em apenas 5 anos; o INPE passa de 100 artigos para 395 artigos, no mesmo período. Entretanto, a variação do IPEN foi mais exponencial, se comparada com as outras duas instituições no quadro geral.

O crescimento das três instituições foi notável a partir de 1983, momento em que o mundo acadêmico começou a dar mais importância aos trabalhos indexados pela WoS, especificamente a partir da década de 1980 (LOUZADA, 2002).

Nesta análise conseguiu-se identificar 2787 autores que foram representados nos 1927 artigos recuperados, sendo autores do IPEN e colaboradores de outras instituições, nos quais, aplicando o modelo de Price (1963) sobre todo o universo, obteve-se um índice de 274 autores que, neste período, configuraram-se como 0 grupo mais produtivo/representativo na somatória total, representando $50 \%$ de toda a produtividade científica. Foi obtido um ranking dos autores do IPEN mais representativos na WoS, porém, é importante que seja destacado que a maioria dos autores dessa instituição teve um índice de publicação significativo, embora esse grupo de autores mais produtivo serve para vislumbrar como foi o comportamento de alguns, no período estudado.

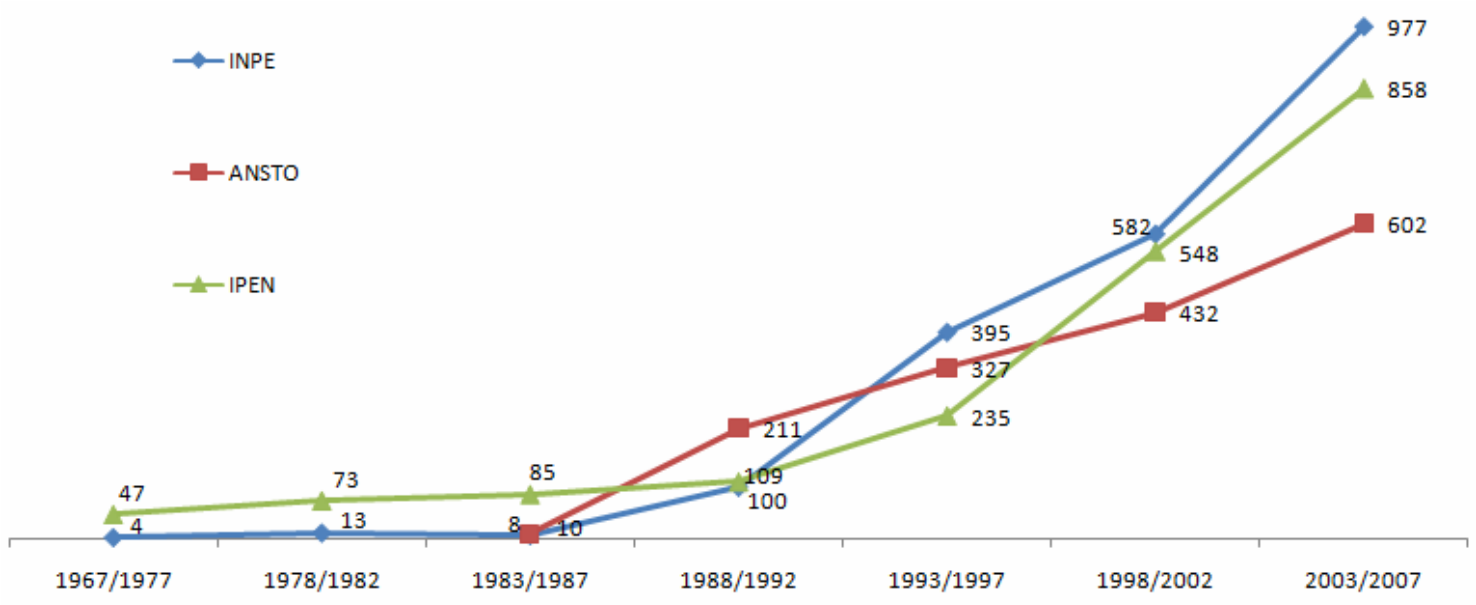

FIGURA 1 - Crescimento científico do IPEN comparativo com o INPE e ANSTO

Fonte: Dados da pesquisa.

Esse tipo de análise também permitiu identificar a elite dos autores, onde aplicando-se a raiz quadrada aos 2787 artigos obteve-se os 52 autores mais representativos, dos quais 49 são pesquisadores com dedicação exclusiva ao IPEN e os outros 3 são credenciados pelo 
Programa de Pós-Graduação, embora não pertençam ao quadro de pesquisadores da instituição estudada.

Neste sentido apresentamos o desempenho desses 52 autores mais produtivos (TAB. 1), ilustrando, assim, o ranking determinante dos autores sem identificação de nomes, somente contemplados pela produtividade do maior ao menor dentro da amostra, agregados ao índice $h$ obtido nas bases da WoS, em 2007.

TABELA 1 Ranking obtido a partir do modelo de Price aplicado aos autores que publicaram pelo IPEN ou em colaboração*

\begin{tabular}{|c|c|c|}
\hline $\begin{array}{l}\text { Ranking dos } \\
\text { autores }\end{array}$ & $\begin{array}{c}\text { İndice de publicação } \\
1964 \text { até } 2007\end{array}$ & $\begin{array}{c}\text { İndice-h relevante } \\
\text { à } 2007\end{array}$ \\
\hline 10 & 112 & 8 \\
\hline $2^{0}$ & 109 & 8 \\
\hline $3^{\circ}$ & 89 & 10 \\
\hline $4^{\circ}$ & 84 & 11 \\
\hline $5^{\circ}$ & 80 & 8 \\
\hline $6^{\circ}$ & 73 & 11 \\
\hline $7^{\circ}$ & 70 & 7 \\
\hline $8^{\circ}$ & 64 & 9 \\
\hline 90 & 57 & 12 \\
\hline $10^{\circ}$ & 56 & 10 \\
\hline $11^{\circ}$ & 53 & 7 \\
\hline $12^{0}$ & 53 & 6 \\
\hline $13^{\circ}$ & 52 & 6 \\
\hline $14^{\circ}$ & 50 & 9 \\
\hline $15^{\circ}$ & 48 & 6 \\
\hline $16^{\circ}$ & 47 & 5 \\
\hline $17^{\circ}$ & 42 & 7 \\
\hline $18^{\circ}$ & 41 & 9 \\
\hline $19^{\circ}$ & 40 & 8 \\
\hline $20^{\circ}$ & 40 & 5 \\
\hline $21^{\circ}$ & 39 & 9 \\
\hline $22^{\circ}$ & 37 & 10 \\
\hline $23^{\circ}$ & 37 & 4 \\
\hline $24^{\circ}$ & 37 & 4 \\
\hline $25^{\circ}$ & 36 & 8 \\
\hline $26^{\circ}$ & 36 & 7 \\
\hline $27^{\circ}$ & 35 & 7 \\
\hline $28^{\circ}$ & 34 & 8 \\
\hline $29^{\circ}$ & 34 & 6 \\
\hline $30^{\circ}$ & 34 & 6 \\
\hline $31^{\circ}$ & 33 & 6 \\
\hline $32^{\circ}$ & 33 & 4 \\
\hline $33^{\circ}$ & 33 & 3 \\
\hline $34^{\circ}$ & 33 & 3 \\
\hline $35^{\circ}$ & 32 & 9 \\
\hline $36^{\circ}$ & 32 & 8 \\
\hline $37^{\circ}$ & 32 & 4 \\
\hline
\end{tabular}




\begin{tabular}{c|c|c}
\hline $38^{\circ}$ & 31 & 5 \\
\hline $39^{\circ}$ & 27 & 7 \\
\hline $40^{\circ}$ & 27 & 3 \\
\hline $41^{\circ}$ & 25 & 9 \\
\hline $42^{\circ}$ & 25 & 5 \\
\hline $43^{\circ}$ & 25 & 1 \\
\hline $44^{\circ}$ & 24 & 6 \\
\hline $45^{\circ}$ & 24 & 5 \\
\hline $46^{\circ}$ & 24 & 5 \\
\hline $47^{\circ}$ & 24 & 4 \\
\hline $48^{\circ}$ & 23 & 3 \\
\hline $49^{\circ}$ & 22 & 6 \\
\hline $50^{\circ}$ & 22 & 2 \\
\hline $51^{\circ}$ & 21 & 9 \\
\hline $52^{\circ}$ & 21 & 6 \\
\hline
\end{tabular}

Fonte: Dados da pesquisa.

Nota: Dados extraídos da WoS no início de 2007, antes da inclusão, nessa base, dos artigos publicados em conferências.

É importante mencionar que esses autores conseguiram manter um índice de visibilidade significativo e contínuo ao longo do período estudado. Foi possível, também, identificar o índice $h$ correspondente ao período de 2007 e efetuar uma comparação desses autores, dada a relevância deste dado entre produtividade e citação dos principais autores.

Em relação ao índice $h$ identificou-se o universo dos mais representativos entre 11 e 12 documentos mais citados. Este dado é relevante porque demonstra o desempenho dos trabalhos dos autores do IPEN na base de dados estudada e demonstra a importância desses agentes para o meio científico da área de energia nuclear, tanto no país como no estrangeiro.

Frente a análise das revistas onde os pesquisadores do IPEN concentram as suas publicações, recuperou-se um número significativo de 435 títulos, todos voltados ao propósito da energia nuclear e ciências relacionadas. Neste segmento, aplicou-se a lei de Bradford, onde identificou-se o primeiro bloco de revistas com um total de 9 títulos (34\% do total de artigos); para o segundo bloco considerou-se 57 títulos (33\% dos artigos); e para o terceiro bloco um total de 369 títulos de revistas (33\% dos artigos).

Na TAB. 2 são relacionadas as revistas do primeiro bloco juntamente com os respectivos fatores de impacto (FI) da JCR. 
TABELA 2 Revistas mais utilizadas segundo o modelo de Bradford com o respectivo FI e a classificação do Qualis/Capes (Engenharias II)

\begin{tabular}{|c|c|c|c|c|c|c|c|c|c|c|}
\hline Revistas & $67 / 78$ & 79/83 & $84 / 88$ & $89 / 93$ & 94/98 & 99/03 & $04 / 07$ & $\Sigma$ & $\begin{array}{c}\text { F.I. } \\
2007\end{array}$ & $\begin{array}{l}\text { Qualis } \\
\text { Capes }\end{array}$ \\
\hline J. Radioanal. Nucl. Chem & & & 15 & 18 & 32 & 45 & 48 & 158 & 0.499 & B1 \\
\hline Radiat. Phys. Chem & 1 & & 1 & & 17 & 46 & 38 & 103 & 0.934 & $\mathrm{~A} 2$ \\
\hline Mater. Sci. Forum & & & & & 2 & 46 & 23 & 71 & ${ }^{*} 0.498$ & B2 \\
\hline Appl. Radiat. Isot & & & & 3 & 11 & 24 & 18 & 56 & 1.008 & A1 \\
\hline $\begin{array}{l}\text { Nucl. Inst. Meth. Phys. } \\
\text { Res }\end{array}$ & & & 4 & 9 & 17 & 18 & 5 & 53 & 1.114 & A1 \\
\hline Radiat. Prot. Dosim & & & 6 & 7 & 5 & 14 & 16 & 48 & 0.528 & $\mathrm{~A} 2$ \\
\hline J. Alloys and Compounds & & & & 3 & 3 & 23 & 8 & 37 & 1.455 & $\mathrm{~A} 1$ \\
\hline Braz. J. Phys & & & & & 1 & 3 & 26 & 30 & 0.478 & B2 \\
\hline Key Eng. Mater & & & & & & 30 & & 30 & ${ }^{\star} 0.278$ & B3 \\
\hline
\end{tabular}

Fonte: Dados da pesquisa.

Notas: $\Sigma$ (Somatória dos documentos nas bases de dados da WoS); F.I. (Fator de Impacto 2007; * 2004). Informações da nova Qualis/Capes.

A revista mais procurada pelos autores do IPEN, na hora de publicar, foi a Journal of Radioanalytical and Nuclear Chemistry, com um percentual $8,92 \%$ de todos os artigos publicados, um número muito significativo se for notado que a área de Energia Nuclear no Brasil interage com vários segmentos, entre eles a engenharia de materiais, química, física e outros, conforme visualizado no primeiro bloco.

O destaque das demais revistas desse bloco foram as revistas Radiation Physics and Chemistry; Nuclear Instruments and Methods in Physics Research e Radiation Protection Dosimetry, percentual acima de $5 \%$ das publicações. Entretanto, o que mais chama a atenção é o grande percentual que as revistas Applied Radiation and Isotopes e Journal Alloys and Compounds adquiriram a partir de 1989, tendo um importante índice crescente produtivo.

Para as revistas relacionadas na TAB. 2, é importante ressaltar o fator de impacto de três revistas com a classificação máxima pelo sistema Qualis/Capes, descritas com A1, sendo as mais importantes para se publicar na área de Engenharia e no segmento estudado (Energia Nuclear).

De modo geral, pode-se afirmar que todas as revistas desse bloco mantiveram um índice significativo, com ressalva aos títulos Materials Science Forum e Key Engineering Materials, que não detêm fator de impacto atualizado, porque passaram a ser consideradas séries monográficas nos últimos anos e, mesmo assim, mantêm o padrão de qualidade em suas publicações, dentro do meio científico.

Dentro desta análise também foi identificado outra quantidade de revistas que não estão relacionadas na TAB. 2, mas que tiveram um índice representativo, das quais destacaram-se: Journal of Magnetic and Magnetisms, Materials Journal, Applied Physics Hyperfine Interactions, Química Nova, Nuclear Instruments Methods Physics Research B, Journal of Non-Crystalline Solids, Physics Review B, Annalsof Nuclear Energy, 
Nuclear Science Engineering, Journal of European Ceramics Society, Journal of Crystalline Growth, Nuclear Instruments Methods Physics Research A, Health Physics, Physics Review C, Brazilian Journal of Medicine and Biological Research, Materials Science Engineering A, Journal of Chromatography A, Journal of Environmental Radioactivity, Journal of Luminescence, Journal of Nuclear Materials, Optical Communications, Toxicon, Materials and Corrosion e Proceedings of the National Academy of Science.

Essas revistas apresentaram uma media de fator de impacto de 1,81 , sendo um índice expressivo dentro de suas respectivas áreas.

$\mathrm{Na}$ análise das relações científicas do IPEN destacou-se a centralidade de alguns autores, com uma representação bem interessante, como o caso de Saiki M, Costa I, Gomes L, Bartolini P, Bressiani JC, Higa OZ, Vieira Junior ND, Lugão AB, Bressiani AHA, Wetter $\mathrm{NU}$, Ribella MTCP, Zezell DM, Morato SP, Rogero SO, Samad RE, Figueiredo AMG, Vasconcellos MBA e Muccillo R. Em se tratando do âmbito institucional, a amplitude da colaboração científica do IPEN é extremamente ampla e com um leque interessante, como veremos na continuação da FIG.2.

Entre os principais parceiros dos autores do IPEN pode-se destacar uma imensa lista de colaboradores, dos quais representamos apenas uma parcela, com as relações mais significativas, como: USP, UNICAMP, UFPE, UNESP, Universidade de Lisboa, UFRGN, Universidade de Coimbra, UFF, UFSC, Gunma University, Tohoku University, University of the Bologna, Uinversidade Nacional de Brasília, University of the Calgary, University of California Riverside, University of the Tennessee, Vanderbilt University, INPE, Instituto Butantã, Instituto de Química Max Planck, Japan Atomic Energy Research Institute, Instituto Superior de Tecnologia e Joint Institute Energy and Nuclear Research. 


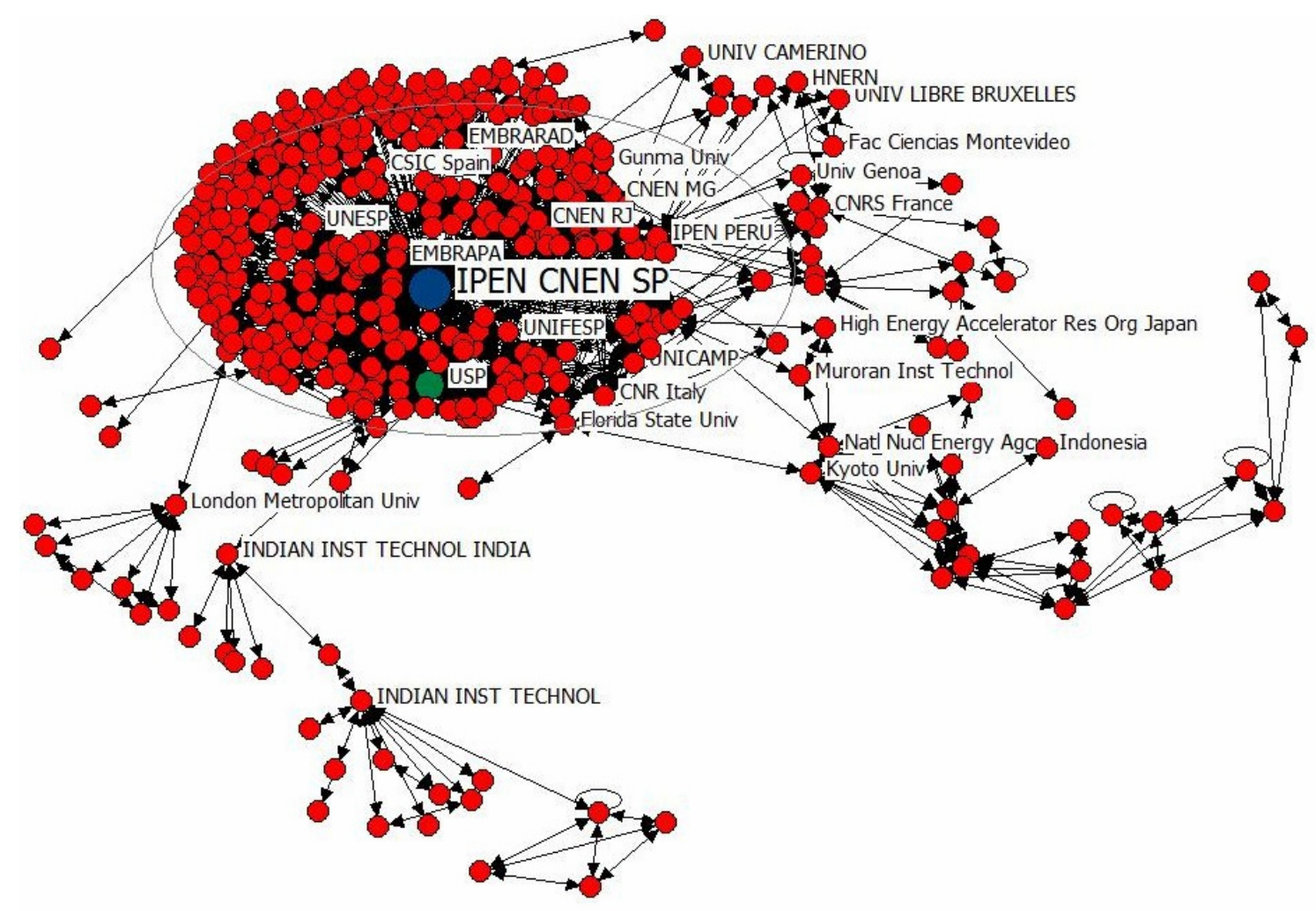

FIGURA 2: Rede social do IPEN

Fonte: Dados da pesquisa.

Nessa análise também é importante destacar as relações com centros de pesquisa e organismos internacionais, como: National Center of Atmospher Research, Centro Brasileiro de Pesquisas Físicas, Centro de Tecnologia Aeroespacial, Centro de Energia Nuclear Agrícola, Federal Research Center Nutricional and Food, IAEA, Hungarian Academy Science e National Academic of Science Belarus. Todas essas relações foram evidenciadas após aplicação do modelo de grafo, no qual foi observada a atuação do IPEN com mais de 1000 instituições espalhadas pelo mundo.

Em relação aos dados sociométricos das cooperações, identificou-se 526 colaborações diretas e 850 de forma indireta, onde a densidade foi relativamente baixa, com um índice de 0,013. O ideal para uma relação bem ampla (como se desenhou na FIG. 2) seria de um número mais perto do algoritmo zero, como por exemplo 0,1 , fato que não ocorreu com as relações nesta análise.

Para o grau de centralidade da rede tem-se como elo principal o IPEN, sendo que na análise geral obteve uma média de 10,42 cooperações. Considera-se que a somatória e a divisão de todos agentes participantes tiveram um índice satisfatório, pois estes dados permeiam entre 10 a 15 cooperações. O IPEN teve muita competência em manter a centralidade e ter também muitas instituições parceiras que exerceram papel de centrais, tudo isso devido ao alto nível de colaboração identificado. 
De modo geral, todos os agentes passam direta ou indiretamente pela sua teia (IPEN), onde quanto maior o número de relações entre outros atores que um indivíduo ou instituição puder intermediar, maior é sua centralidade, e, consequentemente, maior o seu poder e influência sobre a rede (HANNEMAN; RIDDLE, 2005).

Em se tratando da intermediação das relações o índice ficou em 625,03 das 526 relações diretas, partindo do ponto zero que é representado pelo IPEN. Isso mostra como o IPEN exerceu o papel de elo entre outras instituições em todas as análises, pois todas as relações tiveram interferência direta do Instituo de Pesquisas Energéticas e Nucleares.

Dentro das relações diretas também tivemos o índice de vetores, que ficou em 0,6634, que representa uma média entre os elos mais distantes das relações como, por exemplo, a relação entre a Universidade de Camerino e o Instituto de Tecnologia da Índia, que na figura fazem cooperação com o IPEN (elo central), porém não figuram como parceiras entre elas, necessitam de um terceiro elemento para existirem na relação. Neste caso o IPEN atua como uma relação de corrente para unir essas instituições e muitas outras representadas na FIG.2.

\section{Considerações finais}

A partir das análises realizadas concluiu-se que:

- foi possível demonstrar a visibilidade da atividade científica do IPEN, principalmente pelo crescimento exponencial que a instituição apresentou durante o período monitorado, mesmo em comparação com as demais instituições que serviram de parâmetro, INPE e ANSTO;

- houve um salto produtivo significativo a partir da década de 1990, onde a instituição iniciou a geração de trabalhos científicos de qualidade em função da política científica estabelecida, que incentivava além da abertura, a transparência e a divulgação das atividades da área nuclear e áreas correlatas;

- o índice $h$ dos pesquisadores analisados apresentou um resultado significativo, principalmente pela média de citação dos pesquisadores dentro da WoS e pelo fator de impacto das revistas nas quais os pesquisadores publicaram seus trabalhos. Ambos os fatores são indicativos da qualidade da ciência desenvolvida pelos pesquisadores do IPEN;

- no quadro geral, as relações foram bem expressivas e muito amplas, com uma representação forte do IPEN e com seus autores centrais dentro da análise, porém, a densidade da rede não foi satisfatória, enquanto a centralidade foi bem significativa;

- foi possível identificar o grupo de autores mais produtivos, responsável por $50 \%$ de toda a produção científica, embora pertencentes a áreas bastante diversificadas, atuantes dentro do IPEN;

- em relação às revistas utilizadas pelos pesquisadores do IPEN para publicação dos seus artigos, foi possível observar que se tratam de títulos 
de abrangência multidisciplinar, o que confirma a aplicabilidade das técnicas nucleares em várias áreas de atuação. Observa-se também que alguns títulos, embora em pequeno número, são canais de significativa qualidade, dada a média do fator de impacto como: Diabetes (8.261), FASEB Journal (6.791), Clinical Infectious Diseases (6.750), J Cell Sci (6.383), Biomater (6.262), J Nucl Med (5.915), Applied Spectroscopy Reviews (4.250). Esses títulos integraram a $2^{a}$ ou $3^{a}$ zona de dispersão (lei de Bradford);

- devido a área nuclear ser relativamente nova no país e a comunidade científica atuante pequena, se comparada com comunidades de outras áreas, pode-se afirmar que o IPEN tem representatividade significativa na WoS.

Finalmente, para instituições de pesquisas onde a publicação dos resultados decorrentes dessas atividades é um dos principais produtos, é recomendável que um monitoramento como este seja repetido periodicamente. Somente com dados em série é possível efetuar comparações sobre o desempenho da instituição, estabelecer indicadores e fundamentar o estabelecimento de políticas institucionais de produção científica.

\section{Referências}

BATAGELJ, V.; MRVAR, A. Pajek analysis and visualization of large networks. Preprint Series, Ljubljana, v. 41, n. 871, p. 2-26, 2003.

BIOJONE, M. R. Forma e função dos periódicos científicos na comunicação da ciência. São Paulo, 2001. (Dissertação de Mestrado) - Escola de comunicações e Artes, Universidade de São Paulo, 2001.

BENCE, V.; OPPENHEIM, C. Does Bradford: Zipf Law apply to business and management journals in the 2001 research assessment exercise?. Journal of Information Science, Thousand Oaks, v. 30, n. 5, p. 469-474, 2004.

BRADFORD, S. C. Sources of information on specific subject. Engineering: an illustrated weekly journal, Kent, n. 137, p. 85-86, 1934.

CAMPANARIO, J. M. Que importancia tienen los procesos de comunicación em ciencia?. Universidad de Alcalá de Henares, Madrid, España. Projeto financiado pelo Ministerio de Educación, Cultura y Desportos. Disponível em: <http:// www2.uah.es/jmc/webpub/portada.html>. Acesso em: 10 dez. 2004.

CHEN, C. CiteSpace II: detecting and visualizing emerging trends and transient patterns in scientific literature. Journal of the American Society for Information Science and Technology, Maryland, v. 57, n. 3, p. 359$377,2006$.

ESCUDER VALLES, R.; MURGUI IZQUIERDO, S. Introduccíon a la estadística para las Ciencias Sociales. Aravaca: McGraw-Hill, 1995. 428 p. 
FREEMAN, L. C. Centrality in social networks: conceptual clarification. Social Networks, Maryland, n. 1, 1978.

FREEMAN, L. C. Visualizing Social Networks. Journal of Social Structure JOSS, Pittsburgh, v. 1, n. 1, 2000. Disponível em: <http://www.cmu.edu/joss/content/articles/volume1/Freeman.html>. Acesso em: 13 jan. 2010.

GARVEY, W. D. Comunicação: a essência da ciência. Oxford : Pergamon, 1979. $332 \mathrm{p}$.

HANNEMAN, R.; RIDDLE, M. Introduction to social network methods. Riverside: University of California, 2005. 322 p. Disponível em: <http://faculty.ucr.edu/ hanneman/nettext/Introduction_to_Social_Netw ork_Methods.pdf>. Acesso em: 13 jan. 2010.

HIRSCH, J. E. An index to quality and individual's scientific research. Proceedings of the National Academy of Sciences of the United State of American, Melville, v. 102, n. 46, p. 16569-16572, 2005.

INSTITUTO DE PESQUISAS ENERGETICAS E NUCLEARES (IPEN). Relatório de gestão. São Paulo: IPEN-CNEN/SP, 2008.

LOURENÇO, C. A. Automação em bibliotecas: análise da produção via biblioinfo (1986-1994). Revista ACB: Biblioteconomia de Santa Catarina, Florianópolis, v. 2, n. 2, p. 51-63, 1997. Disponível em: <http://revista.acbsc.org.br/index.php/racb/article/view/323/376>.

Acesso em: 13 abr. 2010.

LOUZADA, R. C. R. Sobre o crescimento da produtividade científica brasileira: anotações. Revista de Espaço Acadêmico, São Paulo, v. 2, n. 18, novembro, 2002. Disponível em: < http://www.espacoacademico.com.br/018/18clouzada.htm>. Acesso em: 01 jul. 2010.

MACIAS-CHAPULA, C. A. O papel da informetria e da cienciometria e sua perspectiva nacional e internacional. Ciência da Informação, Brasília, v. 27, n. 2, p. 134-140, 1998.

MICROSOFT. Access Office Professional: Windows Vista. Madrid: Microsoft, 2007a. CD-Rom.

MICROSOFT. Excel Office Professional: Windows Vista. Madrid: Microsoft, 2007b. CD-Rom.

MIRANDA, D. B.; PEREIRA, M. N. F. O periódico cientifico como veiculo de comunicação: uma revisão de literatura. Ciência da Informação, Brasília, v. 25, n. 3, p. 375-382, 1996.

MOURA, E. ITA: avaliação da produção científica (1991-1995). In: WITTER, G. P. (Org.). Produção científica. Campinas: Átomo, 1997.

NIEMINEN, J. On centrality in a graph. Scandinavian Journal of Psychology, Linköping, n. 15, p. 322-336, 1974. 
NORONHA, D. P.; FIGUEIREDO, M. C. F.; ROCHA, M. J. V. Análise bibliometrica da dispersão de artigos sobre saúde publica em periódicos brasileiros. Revista da Escola de Biblioteconomia UFMG, Belo Horizonte, v. 7, n. 1, p. 69-89, 1978.

PINTO, A. L.; RODRÍGUEZ BARQUÍN, B. A. Representação gráfica de indicadores científicos utilizando redes sociais: aplicações válidas para novas investigações no Brasil. In: ENCONTRO NACIONAL DE PESQUISA EM CIÊNCIA DA INFORMAÇÃO (ENANCIB), 6., 2005. Anais... Florianópolis: ANCIB, 2005.

PRICE, D. J. S. Little science, big science. New York: Columbia University, $1963,119 \mathrm{p}$.

PRICE, D. J. S. Hacia una ciencia de la ciencia. Barcelona: Ariel, 1973, 128 p.

SAXTON, M. L. ProCite 5.0. The Library Quarterly, Chicago, v. 71, n. 2, p. 288-290, 2001.

SPINAK. E. Indicadores cienciometricos. Ciência da Informação, Brasília, v. 27, n. 2, p. 141-148, 1998.

TAGUE-SUTCLIFFE, J. An introduction to informetrics. Information Processing \& Management, Maryland, v. 28, n. 1, p. 1-3, 1992.

WITTER, G. P. (Org.). Produção científica. Campinas: Átomo, 1997. 